\title{
Trayectorias y desafíos de la historiografía de los museos de historia natural en América Del Sur
}

\author{
Irina Podgorny ${ }^{1}$ \\ Maria Margaret Lopes ${ }^{2}$
}

RESUMEN: En el siglo XIX los museos de historia natural de América del Sur se constituyeron en instrumentos clave para el intercambio y la circulación de datos y especímenes y, en ese sentido, en loci privilegiados de la infraestructura de las ciencias y del saber. Almacenaron tal cantidad de objetos y colecciones que los organizadores de estas instituciones se enfrentaron al problema de cómo guardarlos y exhibirlos dándoles un orden que pudiera entenderse. Por eso, los museos no pueden separarse de la historia del papel, del archivo y de los catálogos. Este artículo repasa algunas cuestiones de la historiografía producida en las últimas décadas, discutiendo, entre otras cosas, la identificación acrítica entre museos, memoria y representación de la nación. A su vez, propone el desafío de cómo escribir la historia de los museos incorporando los agentes humanos y no humanos y el conjunto de circunstancias que sustentan sus éxitos y fracasos.

PALABRAS CLAVE: Colecciones. Historia de la ciencia. América Latina. Siglo XIX.

ABSTRACT: Powerful tools of interchange and circulation of data and specimens, Natural History museums constituted themselves in several Latin-American countries, such as Argentine and Brazil as privileged loci of epistemic infrastructure since the nineteenth century. The museums gathered huge amounts of collections of surveys of territories and people, always proposed as comprehensive, ultimate and exhaustive endeavors, which made those institutions face the challenges of not only storing and displayng the collections and specimens but also how to order the latter in archives and catalogues that would make them intelligible. Problematizing issues already present in the nowadays consistent literature, including the Latin-American contribution, on museums, the paper discusses among other themes, the acritical identification between museums and the representation of nations and the recurrent notion of museums as place of memory. It proposes as a challenge to the new generation of scholar to ponder how to write these histories incorporating their human and non-human agents as well as the set of events and circunstances that generated their sucesses and failures.

KEYWORDS: Collections. History of science. Latin America. 19th Century.
1. Investigadora Principal del Consejo Nacional de Investigaciones Científicas y Técnicas (CONICET), Prof. Titular ad honorem a cargo del Archivo Histórico - Museo de La Plata, Argentina. E-mail: <ipodgo@isis.unlp. edu.ar>. Este trabajo forma parte del PIP 0176 (CONICET)

2. Professora convidada dos Programas de Pós-Graduação em Política Científica e Tecnológica da Universidade Estadual de Campinas (UNICAMP) e de Ciência da Informação da Universidade de Brasília (UnB), Brasil. E-mail: <mariamargaretlopes@gmail.com>. Este trabajo forma parte del Processo $477134 / 2011-4(\mathrm{CNPq})$. 
3. Cf. Máximo Farro (2009); Irina Podgorny (2007)

4. Cf. Maria Margaret Lopes (1999); Irina Podgorny (2001)

5. Véase Susan Sheets-Pyenson (1988).

6. Cf. Irina Podgorny y Maria Margaret Lopes (2008)

7. Cf. Maria Margaret Lopes (1992); Irina Podgorny (1995)..

8. Cf. Nélia Dias 1991; Paula Findlen (1994); Sophie Forgan (1994); Dorinda Outram (1997); Adalgisa Lugli (1998) y Samuel Alberti (2005).

9. Cf. Maria Margaret Lopes (2001 b).
Hasta hace unos veinte años, la historiografía sobre los museos sudamericanos de historia natural era prácticamente inexistente. Y aunque a veces se los mencionaba entre los establecimientos creados en el desarrollo de las ciencias, abundaban, en cambio, los relatos donde el devenir de estas instituciones se entendía como la obra de alguna figura fundadora y visionaria. En ese tipo de trabajo, los museos se planteaban como sujetos de un relato que los transformaba en una encarnación de la biografía de sus directores y en una empresa de creación de la grandeza nacional. ${ }^{3}$

En ese marco de celebración de la individualidad de los museos y sus fundadores, muy pocos pensaban en las conexiones existentes entre ellos en una escala iberoamericana $y$, mucho menos, transoceánica. ${ }^{4}$ Esto a pesar de la existencia del trabajo de la historiadora estadounidense Susan Sheets-Pyenson ${ }^{5}$, quien había encuadrado al Museo de La Plata y al Museo Nacional de Buenos Aires en la expansión de la llamada "ciencia colonial". Inaugurando una línea de trabajo comparativa, Sheets-Pyenson mostraba las semejanzas que existían entre los museos de Australia, Canadá o la Argentina. En su obra, los museos, sus colecciones y directores se insertaban en una red internacional de intercambio de datos, de publicaciones, de saberes y prácticas ligadas a la promoción de un tipo arquitectónico, de formas de exhibición y organización de las disciplinas científicas. La similitud del mobiliario, las pautas de seguridad, la preocupación por la preservación de los ejemplares y los modos de iluminación parecían repetirse en cada ciudad. Las diferencias surgían, en todo caso, del contenido de las vitrinas. Los museos, incorporados en una red de referencias arquitectónicas y en una lógica de exhibición compartidas, se volvían, en ese sentido, comparables.

Sin embargo, el modelo planteado por Sheets-Pyenson no fue cuestionado; tampoco aceptado. A su publicación se le respondió con silencio, no tanto como resistencia sino como expresión de la clausura y aislamiento que dominaba en el panorama historiográfico de las tradiciones científicas "nacionales": muchas veces a cargo de quienes se consideran sus herederos, estos relatos se referían a un pasado significativo a nivel local, donde ese lado menos excepcional de estos emprendimientos no podía tener cabida. ${ }^{6}$ En esos años marcados por el estudio de las ciencias nacionales y por el desinterés por los museos como objeto de estudio, "Catedrales de la ciencia" fue simplemente ignorado.

Esas discusiones, finalmente, empezaron a aparecer en Brasil y la Argentina. 7 Pero para entonces, el estudio de estas instituciones había ido mucho más allá de los planteos de la llamada historiografía de la "ciencia colonial". Casi de manera paralela, la década de 1990 fue testigo del surgimiento del interés en el orden y los espacios del saber: los gabinetes de rarezas, las colecciones de los objetos más diversos y las sociabilidad urdida a través de la recolección de naturalia y memorabilia en distintos momentos de la historia europea generaron una enorme bibliografía, nuevas revistas y nuevas discusiones. ${ }^{8}$ Los criterios de clasificación, la circulación de imágenes y especimenes, el viaje de campo ; las instrucciones para recolectar y observar mostraron que el museo ocultaba, en 
realidad, un mundo de dimensiones mucho mayores que las comprendidas por las salas de exhibición y los depósitos. ${ }^{10}$

En forma concomitante a la consolidación del estudio de la cultura material y a los diversos giros que la historia de la ciencia fue incorporando a partir de 198511, los nuevos trabajos sobre los museos de historia natural buscaron inspiración en la bibliografía que estaba surgiendo sobre coleccionismo y circulación y formas del conocimiento, donde la microhistoria y la micropolítica institucional jugaban un papel fundamental. ${ }^{12}$

Curiosamente, el mayor impacto de la obra de Susan Sheets-Pyenson no provino de su riqueza historiográfica sino de la sonoridad del título. Subrepticiamente y, quizás, como efecto no deseado, las "catedrales de la ciencia" y, sobre todo, la metáfora de los museos como "templo", hicieron que muchos aficionados a la historia jugaran con ese tópico, enfatizando el aspecto meramente simbólico o representativo y olvidándose de la complejidad que allí se escondía. Muchos hasta creyeron que los museos habían sido creados para ocupar el lugar de las iglesias. Esta línea, promovida por la crítica ideológica de los llamados "estudios culturales" y de los estudios "postcoloniales" se combinó con el furor que en la década de 1990 desencadenaron los trabajos sobre el nacionalismo, la construcción de las tradiciones y la creación de "comunidades imaginadas". ${ }^{13}$ En ese marco, se instaló como lugar común que los museos se establecían como máquinas de representación de la Nación. Nadie, sin embargo, se encargó de demostrar este enunciado. Dejándose impresionar por los edificios monumentales, se creyó en la eficacia de los mismos, repitiendo los tópicos que los creadores usaban al defender la necesidad de construirlos. En esos relatos llenos de juegos de palabras, los museos aparecen como instrumentos del poder y ojos de un Estado que, con un poco de suspicacia y de oficio historiográfico se hubiese descubierto menos fuerte que el argumentado por la lógica de este tipo de trabajos. Más aún, con ellos se reforzaban las conclusiones de la historiografía más tradicional sobre el papel que, en el marco de creación de los estados nacionales, habrían tenido la ciencia y sus instituciones ahora ya no como vehículo del progreso sino de la dominación y consolidación del lado oscuro de la modernidad.

No obstante, esta oleada de trabajos procedentes de marcos conceptuales diferentes, empezó a mostrar la complejidad del tipo de actores involucrados y las tensiones surgidas entre la expansión de un modelo internacional y la emergencia de las instituciones locales. ${ }^{14}$ Asimismo, las nuevas investigaciones revelaron que el modelo de Sheets-Pyenson y la difusión lineal de un modelo europeo, estaba mediado por los que algunos dieron en llamar las "escalas" americanas. ${ }^{15}$ los museos de estas regiones más que inspirarse en los de París, Washington o Londres, surgían en respuesta a las instituciones creadas en ciudades mucho más cercanas. Montevideo, Buenos Aires, La Plata, o Río de Janeiro fueron, para muchos, aún para los científicos europeos radicados en estas regiones, ejemplos más palpables que los transatlánticos. ${ }^{16}$ En esa misma línea, se hacían visibles los intercambios entre los naturalistas e investigadores del hemisferio sur.
10. Cf. Irina Podgorny (2005a).

11. Cf. James Secord (2004).

12. Cf. Tony Bennet (1995).

13. Inspirados por la lectura de Eric Hobsbawm y Benedict Anderson

14. Cf. Achim (2010) y Rodriguez Prada (2010).

15. Cf. Maria Margaret Lopes (2000, 2001a).

16. Cf. Maria Margaret Lopes e Irina Podgorny (2000a; 2000b). 
17. Cf. Maria Margaret Lopes e Irina Podgorny (2009).

18. Cf. Florentino Ameghino (1934, p. 455).

19. Cf. Maria Margaret Lopes y Silvia Figueirôa (2003).

20. Véase Eduardo Alejandro Holmberg (1902, p. 272-3).

21. Cf. Nicolaas Rupke (1994); Claude Schnitter (1996); Máximo Farro (2009) y Susana García (2009).
Interesados en describir la fauna, la flora, el reino mineral y las características de los grupos humanos de la parte austral del continente americano, estos aprendieron que se hacía necesario conocer el estado de la cuestión y las cosas procedentes de las zonas equivalentes de África, Oceanía, Australia y del Océano Índico ${ }^{17}$. Esa circulación permitió, asimismo, pensar qué tipo de museo se quería. Por ello, no llaman la atención los argumentos que Florentino Ameghino (̇ं 18542-1911), Director del Museo Nacional de Historia Natural de Buenos Aires entre 1902 y 1911 , usara ante el gobierno argentino para negociar un nuevo local

por su edificio e instalación actual se encuentra a un nivel más bajo que los museos de provincia, departamentales y municipales de poblaciones o ciudades de último orden. Hasta colonias aisladas en la inmensidad del Pacífico, en los últimos confines del mundo civilizado, como Nueva Zelandia, tienen sus museos instalados en edificios monumentales construidos expresamente. ${ }^{18}$

En la medida que los directores de los museos conocían los progresos de las instituciones de otros lugares del mundo, la historiografía también hubo de dar cuenta de esas dinámicas como mediadoras de la recepción de las ideas y los modelos a seguir: el museo ideal resultaría de la combinación de distintos factores, tal como la cercanía geográfica, la competencia o rivalidad entre ciudades o equipos de trabajo, las afinidades o intercambios con determinados centros metropolitanos. ${ }^{19}$ Y aunque la posesión de "un museo" se equiparaba a un símbolo de civilización y de estar en el mundo de acuerdo con el tono de los tiempos, este argumento más de una vez tomó la forma de un mero tópico. Tal es así que hacia 1900, un viajero, al constatar el estado ruinoso de los museos creados en las provincias argentinas, se preguntaba:

¿Por qué existen? ¿Para qué, si no responden a necesidad alguna? (...) Fundados los museos más por la necesidad de tener iniciativas y hacer algo muy importante, que por otras razones, los gobernantes hallaron en su obra un título digno de honrosa mención, como que a nadie puede decírsele, usted ha hecho mal en darnos un museo. ${ }^{20}$.

Lejos del sentido común que acepta la necesidad de "crear museos", esta reflexión exhibe los desafíos implicados en tal acto. Asimismo, nos recuerda dos cosas: primero, que todas las instituciones para sobrevivir deben negociar permanentemente sus funciones y presupuesto $y$, secundariamente, que existe una gran cantidad de proyectos que no lo lograron. ${ }^{21} Y$ aunque muchos sobrevivieron prometiendo servir ventajosamente a la Patria, los políticos no terminaban de convencerse $y$, en el mejor de los casos, requerían continuas pruebas de la capacidad de adaptación de sus promotores. A la vez, la muletilla sobre la urgencia de un museo fue compartida por políticos, profesionales y aficionados, asociándose a la exploración del territorio y a un fin que parecía no completarse nunca: el conocimiento de las riquezas locales, regionales o nacionales.

Cada uno de estos aspectos no remite a otros tantos: por un lado, a las redes de intercambio y de provisión de datos y artefactos que se tendieron entre 
los científicos y las administraciones de los museos para lograr estos objetivos. La recopilación del material reposó en los modos más diversos: a las exploraciones programadas se sumaron naturalistas viajeros encargados de recoger todo espécimen que se cruzara en el camino y la redacción de instrucciones para los jefes de las oficinas del telégrafo o de los destacamentos de policía. ${ }^{22}$ Enfrentados a funcionar sin presupuesto y sin dinero disponible para el enriquecimiento de las colecciones, los administradores de los museos hicieron de esa falta virtud, desarrollando una enorme ductilidad para el aprovechamiento de las contingencias. No solo ello: una miríada de cazadores, aventureros y oportunistas se dieron cuenta que proveer especimenes a los museos podía colaborar con su propia economía o con la obtención de cierto prestigio como corresponsal. De esta manera, a los depósitos de los museos llegaban por compra o donación cosas recopiladas con objetivos diversos y su propia carga cultural. La historiografía aún nos debe una reflexión acerca de cómo estas cosas procedentes de diversas tradiciones de uso convivieron y aún conviven en un espacio que, supuestamente, las incorporaba a un orden diferente. Momias, plantas, minerales y animales, además de ser especimenes de las ciencias, formaban parte de, por ejemplo, los gabinetes médicos de los charlatanes contemporáneos a los grandes museos. Más aún: muchos vendedores de tónicos y cremas llamaban "museo" al espacio que usaban para atraer pacientes, vendiendo sus colecciones a las grandes "catedrales de la ciencia". ${ }^{23}$ Los objetos, ¿̇perdían en esa transacción el poder medicinal que gozaban en el gabinete del charlatán?. ¿Cómo los entendía quien los había observado en ambos espacios? Estos objetos circulan entre mundos culturales, dinámica en la que se le van adhiriendo significados, que se combinan con los antiguos y van creando nuevos. Sin embargo, todavía faltan estudios sobre estas trayectorias para poder entender que los objetos guardados en los museos están constituidos por diferentes capas culturales, no necesariamente diacrónicas.Los intercambios y circulación de datos y especimenes a veces fueron favorecidos por la afinidad lingüística y cultural de las "comunidades científicas diaspóricas" . 24 Pero en otras circunstancias, esa supuesta comunidad, desencadenó la competencia por el acceso a las fuentes de recursos financieros provenientes de los países de residencia o de origen. ${ }^{25}$ Alemanes, franceses, estadounidenses, sardos, portugueses y "nacionales" se aliaron pero también desarmaron alianzas en función de los intereses que surgían del estudio de la ciencia ${ }^{26}$ y la necesidad de supervivir en América. En un medio percibido y vivido como lábil y hosti 27 , donde la continuidad de los proyectos iniciados nunca estaba asegurada, los rimbombantes discursos, las alianzas y las guerras científicas estuvieron a la orden del día entre. Sin embargo, estas idas y venidas no evitaron la intensa movilidad de especimenes, libros y cartas por distintos medios de transporte y comunicación, permitidos también por los vínculos comerciales entre los museos y las casas proveedoras radicadas en uno u otro país. ${ }^{28}$

Estos problemas nos remiten, asimismo, al papel que los museos de historia natural adoptaron en países como la Argentina y Brasil: un locus privilegiado
22. Cf. Andrea Pegoraro (2009); Irina Podgorny y Wolfgang Schäffner (2000) y Marie-Noëlle Bourguet (2008).

23. Cf. Irina Podgorny (2009b, 2012, 2013).

24. Véase H. Glenn Penny (2002).

25. Cf. Irina Podgorny (2009a).

26. Cf. Irina Podgorny (2001; 2005b).

27. Cf. Hebe Vessuri (1995).

28. Cf. José Antonio Pérez Gollán (1995) y Susana García (2009). 
29. Cf. Maria Margaret Lopes (2009).

30. Cf. Maria Margaret Lopes y Alda Heizer (2011).

31. Cf. William Flinders Petrie (1904).

32. Véase Irina Podgorny (2008).

33. Cf. Adolph Bernard Meyer (1901, p. 7). para la producción del conocimiento. ${ }^{29}$ En ese sentido, más allá del potencial estudio de los recursos económicos disponibles, los museos se armaron como el repositorio de la evidencia y de las observaciones de las distintas disciplinas científicas que, en muchos casos, se creaban o consolidaban alrededor de las colecciones. ${ }^{30}$ Estos museos de fines del siglo XIX amasarían grandes colecciones que permanecerían sin catalogar. En ese sentido, el supuesto inventario quedaba en un horizonte cada vez más lejano. Los museos más que ordenar los recursos del territorio, quedaron ałados a una dinámica de saturación compulsiva. Los exitosos, crecían pero sin rumbo, ligados a los regalos, al aprovechamiento de las circunstancias y a lo imprevisto. No siempre dotados de una fuerza de trabajo remunerada para registrar las entradas de los objetos, quedaron imposibilitados para procesar los datos en forma de inventarios. Paradóiicamente, los museos movilizaron volúmenes de materiales desde espacios lejanos a su emplazamiento urbano pero no siempre pudieron garantizarles un nuevo orden que permitiera su uso o estudio. Esta situación que se repetía en Berlín, La Plata, Buenos Aires y Londres, llevó a Flinders Petrie ${ }^{31}$ a definir a los museos de inicios del siglo XX como fantásticos "osarios de pruebas asesinadas". ${ }^{32}$

Por eso mismo para los encargados de resolver el problema de los museo de 1900, la solución no residiría en sus salas o arquitectura sino en el catálogo y la creación de un cuerpo de burócratas dedicados a la enorme tarea de registrar cada uno de los movimientos de los objetos que debían entrar en el museo para no volver a salir. El modelo ideal parecía ser el del Field Museum de Chicago donde en el archivo se conservaban cada uno de los documentos originales de las colecciones, cada una con un número fijo que los identificaba. Cada acta solo se movería contra entrega de un recibo de uno de los funcionarios de los distintos departamentos. Este movimiento se registraba en libros y fichas: hasta octubre de 1900 existían 94 tomos de catálogo con un total de 215.000 entradas, a los que se sumaban 75.000 fichas o tarietas. ${ }^{33}$ Todo se registraba en unos finos cartones de un tamaño determinado, llamados cards por lo que los catálogos adoptaban el nombre de card-catalogue. Se podía saber inmediatamente de qué lugar del planeta, coleccionista, mecenas o vendedor procedían las colecciones. A través del número de entrada podría llegarse a las actas (listas, correspondencia), al libro de inventario o al fichero (descripción, localidad). Por otro lado, el número de cada ejemplar conducía directamente al libro de inventario, - gracias al número de ingreso, se llegaba a la correspondencia de las actas. Solo de esta manera, la colección se podía constituir verdaderamente en un todo. Este complejo sistema de inventario y de registro le daba sentido a las colecciones del siglo que se iniciaba. El recorrido científico por las colecciones del museo no se hacía en las salas sino a través del registro en las fichas. Para ello se necesitaba una increíble cantidad de trabajo burocrático a alto costo para el empleador. Sin embargo, el mismo se compensaba por el ahorro de tiempo que este orden provocaba. Este sistema generaba, a su vez, nuevas necesidades. Entre ellas, la producción de etiquetas, negativos, impresiones, imágenes luminosas. El museo 
moderno surgiría, en realidad, de esta combinación entre la antigua cultura europea de los secretarios ${ }^{34}$ y la administración de los objetos de los grandes almacenes americanos, tal como la impuesta en el Field Museum de Chicago. Como recordó Secord ${ }^{35}$, aún queda por hacerse una historia de la ciencia que recupere ese orden dado por los catálogos e inventarios de museos y colecciones, de alguna manera, la verdadera clave del orden dado a las cosas.

Por otro lado, la ubicación del museo en la ciudad, las grandes salas de exhibición y los edificios monumentales, cobraron para los científicos un valor simbólico y político. Representaron su capacidad de negociación y las alianzas tejidas para conseguirlos, no como necesidad para su trabajo cotidiano sino como monumentos a su capacidad de gestionar recursos para el desarrollo de disciplinas hasta entonces encarriladas privadamente. Por eso, a pesar del auge de los últimos años por la comprensión de los museos como "lugar de la memoria", los museos de historia natural no parecen cuadrar en esa categoría. El uso de las exhibiciones para la enseñanza científica universitaria, un espacio que, aparentemente, estuvo dedicado casi con exclusividad al público general permanece como un tema pendiente de la investigación. Poco a poco, los científicos irían abandonando hasta su interés en las mismas, apareciendo nuevas profesiones encargadas exclusivamente de su cuidado y diseño. La práctica de la ciencia se refugiaría en los laboratorios, los depósitos y las clases universitarias, lejos de la mirada pública. Sin un uso en la investigación de los materiales expuestos, el museo - como institución continuaría actuando como vitrina y espacio de representación de la ciencia.

El gran museo decimonónico pudo haber surgido como necesidad de disputar nuevos nichos para estas nuevas prácticas científicas y, sobre todas las cosas, para lograr los favores y la protección económica del Estado. En la Argentina, como en Inglaterra y en otros países, tales iniciativas, para concretarse, necesitaron de individuos flexibles a los rumbos de la política. Quienes supieron entretejer su prestigio personal y sus redes sociales con los supuestos intereses de la Nación lograron llevar adelante dichos proyectos. En el caso argentino, la labilidad del Estado complica la historia aún más y quizás nos sugiera explorar con más cuidado ciertos lugares comunes sobre la alianza entre "la ciencia", "el poder" y "el control estatal".

Y aquí surge un problema: si el Estado y la Nación no son los sujetos que empujan la creación de museos, ¿̇cómo evitar una historiografía que se centre en la voluntad de esos artífices de su supervivencia?. ¿Cómo evitar contar la historia de los museos tomando a los fundadores como centro, o peor aún, como autor? Porque más allá de la ilusión biográfica, tampoco se trata de una situación idéntica a la de la modernidad inicial, cuando la identidad del coleccionista estaba íntimamente ligada a su colección. ${ }^{36} \mathrm{El}$ marco del nacionalismo o entender al museo como la herramienta del Estado permitiría salvar este aspecto pero, sin embargo, los datos concretos atentan contra ese argumento. Como en todas las instituciones, científicas o no, en los museos más que la macropolítica rige la política del poder de las relaciones urdidas en el seno de las mismas; la contingencia de los
34. Cf. Bernard Siegert y Joseph Vogl (2003).

35. Véase James Secord (2004).

36. Cf. Paula Findlen (1994). 
acontecimientos y los automatismos de las acciones y del discurso. Sin dudas, es un desafío para las nuevas generaciones pensar cómo escribir estas historias sin un sujeto claro que o, dicho de otra manera, hacerlo incorporando los agentes humanos y no humanos y las constelaciones de acontecimientos y circunstancias que sostienen sus éxitos y fracasos.

\section{REFERÊNCIAS}

ACHIM, Miruna. Setenta pájaros africanos por antigüedades mexicanas: canjes de objetos y formación del Museo Nacional de México (1825-1867). L'Ordinaire Latino-américain. Toulouse, n. 212, p.13-32, 2010.

ALBERTI, Samuel. Objects and the Museum. Isis, Bruselas, v. 96, n. 4, p. 559-571, dec.2005.

AMEGHINO, Florentino. Informe elevado al Señor Ministro de Justicia e Instrucción Pública, por el Director del Museo Nacional de Historia Natural, sobre el desastroso estado actual de este establecimiento. In: . Obras Completas y Correspondencia Científica, v. 18. La Plata: Taller de Impresiones Oficiales, 1934, p. 439-522.

BENNETT, Tony. The Birth of the Museum: History, Theory, Politics. London; New York: Routledge, 1995.

BOURGUET, Marie-Noëlle. Escritura del viaje y construcción científica del mundo. La libreta de Italia de Alexander von Humboldt. Redes, Quilmes, n. 28, p.81-95, 2008.

DIAS, Nélia. Le Musée d'Ethnographie du Trocadéro (1878-1908). Anthropologie et Muséologie en France. Paris: CNRS, 1991.

FARRO, Máximo Ezequiel.La formación del Museo de La Plata. Coleccionistas, comerciantes, estudiosos y naturalistas viajeros a fines del siglo XIX. Rosario: Prohistoria Ediciones, 2009.

FINDLEN, Paula. Possessing Nature. Museums, Collecting, and Scientific Culture in Early Modern Italy. Berkeley: University of California Press, 1994.

FORGAN, Sophie. The Architecture of Display: Museums, Universities, and Objects in Nineteenth-Century Britain. History of Science, Bucks, v. 32, n.96, p. 139-162, jun.1994.

GARCIA, Susana. Enseñanza científica y cultura académica. La Universidad de La Plata y las Ciencias Naturales (1900-1930). Rosario: Prohistoria, 2009.

HOLMBERG, Eduardo Alejandro. Museos provinciales y museos regionales. Anales de la Sociedad Científica Argentina, Buenos Aires, v. 53, p. 271-274, 1902.

LOPES, Maria Margaret. Brazilian Museums of Natural History and International Exchanges in the transition to the 20th Century. Boston Studies in the Philosophy of Science, Dordrecht, v. 136 , p. $193-200,1992$.

Fósseis e museus na Argentina e no Brasil, no século XIX: uma contribuição à história da Paleontologia na América Latina. Asclepio, Madrid, v. 22, n.2, p. 145-164, 1999. 
Cooperação científica na América Latina no final do século XIX: os intercâmbios dos museus de ciências naturais. Interciencia, Caracas, v. 25, n.5, p. 228-233, 2000.

A mesma fé e o mesmo empenho em suas missões científicas e civilizadoras: os museus brasileiros e argentinos do século XIX. Revista Brasileira de História, São Paulo, v. 21, n. 41, p. 55-76, 2001a. Disponível em: <www.scielo.br/pdf/rbh/v21n41/a04v2141.pdf>.

. Viajando pelo campo e pelas coleções: aspectos de uma controvérsia paleontológica. História, Ciência, Saúde - Manguinhos, Rio de Janeiro, v.8, suplem., p. 881-897, 2001 b. Disponível em: <http://dx.doi.org/10.1590/S0104-59702001000500005>.

. O Brasil descobre a pesquisa científica. Os Museus e as ciências naturais no século XIX, 2 a ed.. São Paulo: Hucitec; Brasília: UnB, 2009. [1997]

LOPES, Maria Margaret; FIGUEIRÔA, Silvia Fernanda de Mendonça. A criação do Museu Paulista na correspondência de Hermann von Ihering (1850-1930). Anais do Museu Paulista: História e Cultura Material, São Paulo, v.10-11, n.1 p. 23-35, 2003. Disponível em <http:// www.scielo.br/scielo.php?script=sci_arttext\&pid=S0101-47142003000100003\&lng=en\&nrm=i so>.ISSN0101-4714>.

LOPES, Maria Margaret; HEIZER, Alda L.(coord.). Colecionismo, práticas de campo e representações. Campina Grande: EDUEPB, 2011.

LOPES, Maria Margaret; PODGORNY, Irina. Caminos cruzados: el Museo Nacional de Historia Natural de Montevideo en la documentación del Museo Nacional de Buenos Aires. Ciencia Hoy, Buenos Aires, v. 10, n.57, p. 15-20, 2000a.

The Shaping of Latin American Museums of Natural History, 1850-1990. Osiris, Chicago, v. 15, p. 108-118, 2000b.

Paleontologia e evolucionismos no sul da América: a biogeografia de Wallace como um dos obstáculos centrais para interpretar a história geológica do hemisfério sul. In: DOMINGUES, Heloisa. M. B.; SÁ, Magali R; PUIG-SAMPER, Miguel A.; RUIZ GUTIERREZ, Rosaura. (org.). Darwinismo, meio ambiente, sociedade. São Paulo: Via Lettera; Rio de Janeiro: MAST, 2009, p. 143-160.

LUGLI, Adalgisa. Naturalia et Mirabilia. Les cabinets de curiosités en Europe. Paris: Adam Biro, 1998. [1983]

MEYER, Adolph Bernhard. Über Museen des Ostens der Vereinigten Staaten von Nord Amerika. Berlin: Friedländer \& Sohn, 1901.

OUTRAM, Dorinda. New Spaces in Natural History. In: JARDINE, Nicholas et al. (org.). Cultures of Natural History. Cambridge, Cambridge University Press, 1997, p. 249-65.

PEGORARO, Andrea. Las colecciones del Museo Etnográfico de la Universidad de Buenos Aires: un episodio en la historia del americanismo en la Argentina, 1890-1927. Tesis (Doctorado), Facultad de Filosofía y Letras de la Universidad de Buenos Aires, Buenos Aires, 2009.

PENNY, H. Glenn. Objects of Culture: Ethnology and Ethnographic Museums in Imperial Germany. Chapel Hill: The University of North Carolina Press, 2002.

PÉREZ GOLLÁN, José Antonio. Mr. Ward en Buenos Aires: los museos y el proyecto de nación a fines del siglo XIX. Ciencia boy, Buenos Aires, v. 5, n. 28, p. 52-58, 1995.

Annals of Museu Paulista. v. 21. n.1. Jan.-Jun. 2013. 
PETRIE, William Matthew Flinders. Methods and Aims in Archaeology. Londres: Macmillan, 1904.

PODGORNY, Irina. De Razón a Facultad: funciones del Museo de La Plata en el período 1890-1920. Runa, Buenos Aires, v. 22, p. 89-104, 1995.

El camino de los fósiles: las colecciones de mamíferos pampeanos en los museos franceses e ingleses. Asclépio, Madrid, v. 53, n.2 , p. 97-116, 2001.

. La mirada que pasa: museos, educación pública y visualización de la evidencia científica. História, Ciências, Saúde-Manguinbos, Rio de Janeiro, v. 12, suplem., p. 231-264, 2005a.

. Bones and Devices in the Constitution of Palaeontology in Argentina at the End of the Nineteenth Century. Science in Context, Cambridge, v. 18, n. 2, p. 249-283, 2005 b.

Embodied Institutions. La Plata Museum as Francisco P. Moreno's Autobiography. In: VALENTE, Maria Esther A.(ed.). Museums of science and technology - Interpretations and Activities to the Public. Rio de Janeiro: MAST, 2007, p. 95-103.

La prueba asesinada. El trabajo de campo y los métodos de registro en la arqueología de los inicios del Siglo XX. In: LÓPEZ BELTRÁN, Carlos y GORBACH, Frida (coord.). Saberes locales. Ensayos sobre historia de la ciencia. Zamora: El Colegio de Michoacán, 2008, p. 169-205.

El sendero del tiempo y de las causas accidentales. Los espacios de la antigüedad del hombre en el Plata, 1850-1910. Rosario: Prohistoria, 2009a.

La industria y laboriosidad de la República. Guido Bennati y las muestras de San Luis, Mendoza y La Rioja en la Exposición Nacional de Córdoba. In: LLUCH, Andrea.; DI LISCIA, Maria Silvia (ed.). Argentina en exposición. Ferias y exhibiciones durante los siglos XIX y XX. Madrid-Sevilla: CSIC, 2009b, p. 21-59.

Charlatanes. Crónicas de remedios incurables. Buenos Aires: Eterna Cadencia, 2012. Colección Nuestra América.

Travelling Collections in Nineteenth-Century Latin America. Museum History Journal, Leeds, v. 6, n. 2, p. 127-146, jul.2013.

PODGORNY, Irina.; LOPES, Maria Margaret. El desierto en una vitrina, Museos e historia natural en la Argentina del Siglo XIX. México: Limusa, 2008.

PODGORNY, Irina; SCHÄFFNER, Wolfgang. "La intención de observar abre los ojos". Narraciones, datos y medios técnicos en las empresas humboldtianas del siglo XIX. Prismas, Buenos Aires, v. 4, p. 217-27, 2000.

RODRIGUEZ PRADA, Maria Paola. La création du Musée national de Colombie (1823 1830). L'influence scientifique d'un modèle français. Tesis (Doctorado en Historia de l'Arte), Université Paris 1 Pantheon-Sorbonne, Paris, 2010.

RUPKE, Nicolaas. Richard Owen. Victorian Naturalist. New York: Yale University Press, 1994. SECORD, James A. Knowledge in Transit. Isis, Bruselas, v. 95, n.4, p. 654-72, dec. 2004. 
SCHNITTER, Claude. Le développement du Muséum national d'histoire naturelle de Paris au cours de la seconde moitié du XIXe siècle; "se transformer ou périr". Revue d'histoire des sciences, Paris, v. 49, n. 1, p. 53-97, 1996.

SHEETS-PYENSON, Susan. Cathedrals of Science. The Development of Colonial Natural History Museums during the Late Nineteenth Century. Montreal: McGill-Queen's University Press, 1988.

SIEGERT, Bernard.; VOGL, Joseph (ed.). Europa. Kultur der Sekretäre. Berlín: Diaphanes, 2003.

VESSURI, Hebe. El Crecimiento de una Comunidad Científica en Argentina. Cadernos de História e Filosofia da Ciencia, Campinas, s.3, v. 5, n.esp., p. 173-222, 1995. 